Cahiers « Mondes anciens »

ANCIENS

Histoire et anthropologie des mondes anciens

15 | 2022

Les parures divines

\title{
Membra disjecta? Réflexions sur la matérialité des coiffes divines de Mésopotamie archaïque
}

Membra disjecta? Some Remarks on the Materiality on the Divine Headresses

of Early Mesopotamia

\section{Catherine Breniquet}

\section{(2) OpenEdition}

\section{Journals}

Édition électronique

URL : https://journals.openedition.org/mondesanciens/3808

DOI : 10.4000/mondesanciens.3808

ISSN : 2107-0199

Éditeur

UMR 8210 Anthropologie et Histoire des Mondes Antiques

Référence électronique

Catherine Breniquet, «Membra disjecta ? Réflexions sur la matérialité des coiffes divines de Mésopotamie archaïque », Cahiers « Mondes anciens » [En ligne], 15 | 2022, mis en ligne le 18 janvier 2022, consulté le 21 janvier 2022. URL : http://journals.openedition.org/mondesanciens/3808 ; DOI : https://doi.org/10.4000/mondesanciens.3808

Ce document a été généré automatiquement le 21 janvier 2022.

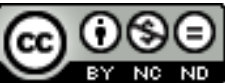

Les Cahiers «Mondes Anciens » sont mis à disposition selon les termes de la licence Creative Commons Attribution - Pas d'Utilisation Commerciale - Pas de Modification 4.0 International. 


\title{
Membra disjecta? Réflexions sur la matérialité des coiffes divines de Mésopotamie archaïque
}

\author{
Membra disjecta? Some Remarks on the Materiality on the Divine Headresses \\ of Early Mesopotamia
}

Catherine Breniquet

\section{Introduction}

1 Parce qu'elle porte le regard, la tête est sans doute le premier élément (avec la silhouette) par lequel on appréhende un individu (Coquet 1994). Regard, cheveux, sourire, maquillage participent ainsi à l'alchimie souvent mystérieuse des relations humaines qu'elles soient de domination, acceptées ou subies, ou de séduction. Tous les peuples, sans doute depuis des origines bien lointaines, en ont fait l'expérience (Le Breton 1992). En outre, la tête est susceptible de devenir le support d'attributs divers ou de parures : bijoux, bandeaux, tiares, etc. qui matérialisent cette fois spécifiquement, comme tous les autres éléments d'ornementation corporelle, l'identité sociale, humaine ou divine, de celui qui les porte, ainsi qu'une protection dont l'efficacité est toute magique et sans lien avec la nature des parures elles-mêmes. La Mésopotamie n'échappe pas à cette règle et l'on rappellera à cette occasion combien Ishtar, se dépouillant de tous ses bijoux et attributs entre, vulnérable, aux Enfers, avec le risque de ne pas en revenir (Contenau 1952, p. 214).

2 Pour qui croyait aux vertus de l'introduction d'une perspective anthropologique en archéologie, une exposition très spectaculaire organisée au musée de la Fondation Dapper à Paris avait alerté la communauté des scientifiques et des amateurs d'art premier sur l'extraordinaire inventivité des peuples africains en matière de coiffures, terme entendu ici dans une acception large (Falgayrettes-Leveau 2003). Recherche esthétique, symbolique des arrangements, complexité des coiffes et simplement (mais rien n'est simple en ce domaine) matérialité des éléments rapportés sur la tête humaine 
démontraient le caractère polysémique de ces ornements. La perspective offerte partait de l'Égypte ancienne pour s'achever sur les grandes civilisations du centre et ouest de l'Afrique. Ethnographique, historique et sociale tout à la fois, cette exposition offrait aussi l'intérêt de confronter la ou les réalités à leurs représentations sculptées et donc, d'alimenter une réflexion transversale.

3 La table ronde organisée par l'UMR 8210 ANHIMA et le laboratoire d'excellence Hastec sous l'égide de Florence Gherchanoc et Louise Quillien ${ }^{1}$ a offert l'opportunité de traiter la question, non pas tant du point de vue des mortels que de celui des dieux. Dans l'Orient ancien, on sait combien nos sources sont paradoxalement foisonnantes lorsqu'il s'agit des textes évoquant les dieux, et maigres, lorsqu'on en vient à rechercher la dimension matérielle des parures divines à travers l'archéologie. Les incessants pillages et recyclages des matériaux nous obligent à contourner la difficulté liée à leur absence en tentant de trouver dans l'iconographie l'image du réel. L'exercice est doublement délicat. L'identification de ce qui est montré est souvent difficile en raison des dimensions des objets, de leur état de conservation ou des conventions artistiques. Quant à la question du rapport entre signifiant et signifié, elle se heurte aux limites de notre connaissance, notamment pour les périodes où les textes sont peu informatifs, au moment où l'écriture est introduite par exemple. En effet, nous ne connaissons quasiment aucune statue divine avérée et complète pour toute l'histoire de l'Orient ancien (Spycket 1968). Les matériaux constitutifs de ces statues, notamment du bois recouvert de placages en métal précieux, y sont sans doute pour beaucoup : le bois disparaît, le métal est recyclé, les incrustations en pierres semi-précieuses sont récupérées, etc. Seule, la statue akkadienne en pierre de Narundi (ca. 2100 av. J.-C.), découverte à Suse et partiellement conservée, fait exception (Amiet 1976, p. 38-39). Les parures, quant à elles, généralement dissociées de leur support, se résument à quelques fragments épars, n'offrant qu'un pâle reflet de leur splendeur passée... Les difficultés culminent pour les périodes historiques les plus anciennes de Mésopotamie, comme les époques d'Uruk (ca. 3900-3100/3000) et protodynastique (ca. 2900-2340), encore documentées de façon insuffisante.

4 L'histoire de l'Orient ancien couvre treize millénaires, des débuts de la sédentarisation qui ouvrent le Néolithique, jusqu'à la conquête de l'Orient par Alexandre en 333 av. J.-C. La démesure géographique est également de mise, d'Istanbul à Kabul et de la mer Noire au Yémen! Si l'on réduit à la Mésopotamie (Irak et Syrie) le propos, la permanence d'un certain nombre de traits culturels, comme la polarité temples/palais, a été pointée de longue date (Oppenhein 1970). Ce monde est interconnecté tant dans l'espace que dans le temps. Mais une telle situation autorise-t-elle pour autant les interprétations rétrospectives à partir de faits et de textes plus récents, gommant ainsi toutes les phases formatives et tous les changements de sens? On peut en douter. Un point final radical est mis au fonctionnement initial de ces civilisations de basse Mésopotamie avec la conquête et l'unification par Sargon d'Akkad vers 2340 av. J.-C. au sein d'un premier empire territorial d'envergure abolissant les liens de dépendance entre individus remontant à la "préhistoire" récente de la Mésopotamie (Huot 2005). L'époque d'Akkad voit par ailleurs la réorganisation et la rationalisation du répertoire iconographique divin, avec des attributs plus clairement fixés (Amiet 1977). Ce sont donc la cohérence originelle du monde mésopotamien pré et protodynastique, une approche de l'iconographie qui n'est pas qu'illustrative, et sur la nécessité d'explorer «l'agency » divine (Gell 1998) qui fondent le présent travail. 


\section{Premières représentations de divinités}

De cette longue histoire de l'Orient ancien, la tradition académique a retenu que les divinités portaient des coiffures cornues, des diadèmes, des caches-cheveux, des casques, des tiares à un ou plusieurs rangs de cornes (Black et Green 1992, p. 93 et 102-103). Toutefois, la diversité est grande en fonction des époques. La formule a été déclinée en multiples variantes dont témoigne la classification largement diachronique de Boehmer (1967 et 1971, p. 416). Ne connaissant guère la contrepartie matérielle de ces représentations, il est difficile de savoir dans quelle mesure il s'agit de réalités tangibles, prises dans une véritable évolution chronologique ou de simples jeux iconographiques archaïsants. La question n'est pas anodine car si l'on s'accorde sur le fait que l'apparition des premières divinités est solidaire de celle de la ville, de l'écriture, de la royauté et d'une forme d'état territorial (Forest 1996), il n'existe pourtant aucune représentation claire des premières divinités poliades qui pourrait inaugurer la série. Les sanctuaires, eux, sont bien évoqués dans les textes archaïques d'Uruk (Englund 1998, p. 102), de même que le nom de la déesse Inanna (Collins 1994 ; Asher-Greve et Westenholz 2013, p. 39), mais l'absence de monuments inscrits et les découvertes, qui sont toujours en contexte secondaire, ne permettent pas de lever l'ambiguïté venant des objets eux-mêmes ${ }^{2}$. Le cas le plus emblématique demeure celui de la « Dame d'Uruk » (Nöldecke et Lenzen 1940, pl. 1 ; Becker 1993, pl. 65 à 68) (fig. 1).

Fig. 1 : Masque en pierre grandeur nature dit "Dame d'Uruk".
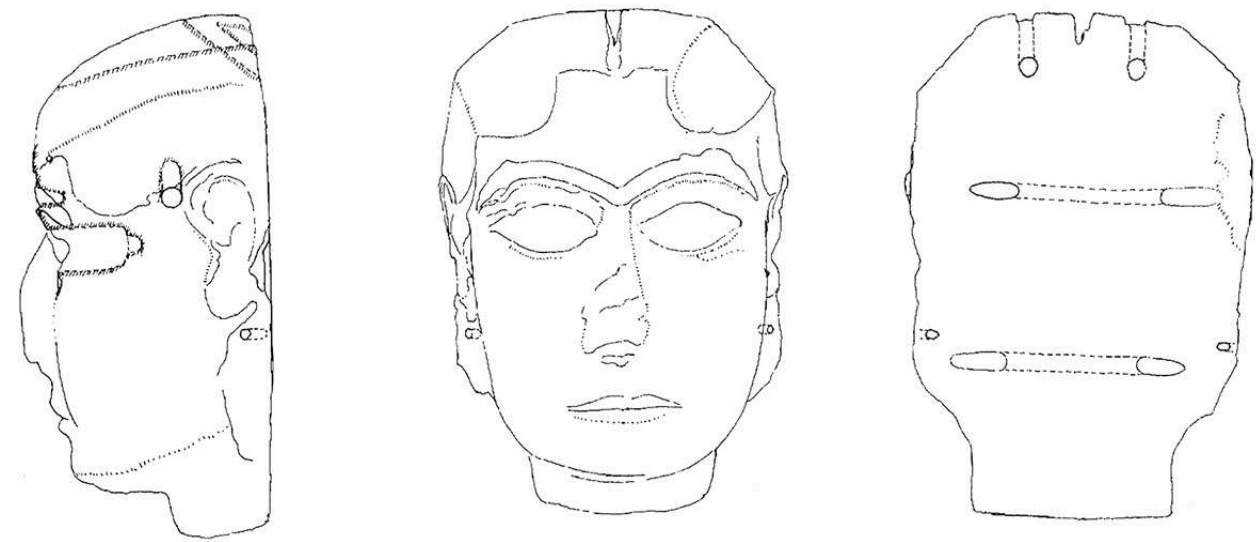

Hauteur : 20 cm. Uruk, ca. 3000 av. J.-C. Musée de Bagdad.

D'après Nöldeke et Lenzen 1940, pl. 1.

Masque plein sculpté en haut relief dans le marbre, datant des environs de 3000 av. J.C., l'objet évoque un visage féminin au style sévère, grandeur nature, qui pourrait bien être celui de la première statue divine jamais réalisée (Forest et Gallois 2006, p. 23). Plusieurs caractéristiques en conforteraient cette lecture : le matériau d'exception, la dimension humaine du visage qui tranche avec tout ce qui est connu en matière de statuaire de l'époque d'Uruk, l'idéalisation du visage, le fait que cette demi-tête ait été initialement rapportée sur une forme-support aujourd'hui disparue, peut-être en bois, comme en témoignent les mortaises visibles sur l'envers, les incrustations sans doute en pierre semi-précieuse des yeux et des sourcils, les ornements métalliques rapportés 
disparus également sur la tête et les cheveux, et aux oreilles. La tête appartenait donc à un ensemble composite qui pourrait parfaitement correspondre aux descriptions textuelles des statues divines ultérieures (Spycket 1968). Toutefois, les éléments déterminants ont disparu, qu'ils aient été refondus, recyclés ou réutilisés. Le contexte secondaire de découverte, une favissa, n'autorise guère une extrapolation assurée. Le caractère unique de cet objet exceptionnel par sa qualité interdit, de fait, toute comparaison. Nous n'avons ainsi qu'un faisceau d'indices convergents.

7 Il n'en va pas de même au Protodynastique II et III (ca. 2800-2340 av. J.-C.). Vases en pierre, stèles, plaques perforées, auxquels il faut ajouter les sceaux-cylindres sont les supports d'une iconographie officielle raisonnablement homogène sur l'ensemble de la sphère d'influence sumérienne (Mésopotamie et Syrie), impliquant les représentants du pouvoir et les dieux dans des scènes de libation, de banquet, de combat, etc. Parmi les exemplaires les plus remarquables, on citera le fragment de relief AO 48 du musée du Louvre, le fragment du vase représentant la déesse Nisaba conservé au musée Pergame de Berlin, ou encore la stèle des Vautours du Louvre et les plaques perforées. Sans être uniformes, ces représentations montrent une ébauche de standardisation absente des époques antérieures (fig. 2). On repère les divinités à leur position, à leur coiffure cornue et à leurs attributs.

Fig. 2a : Bas-relief protodynastique fragmentaire.

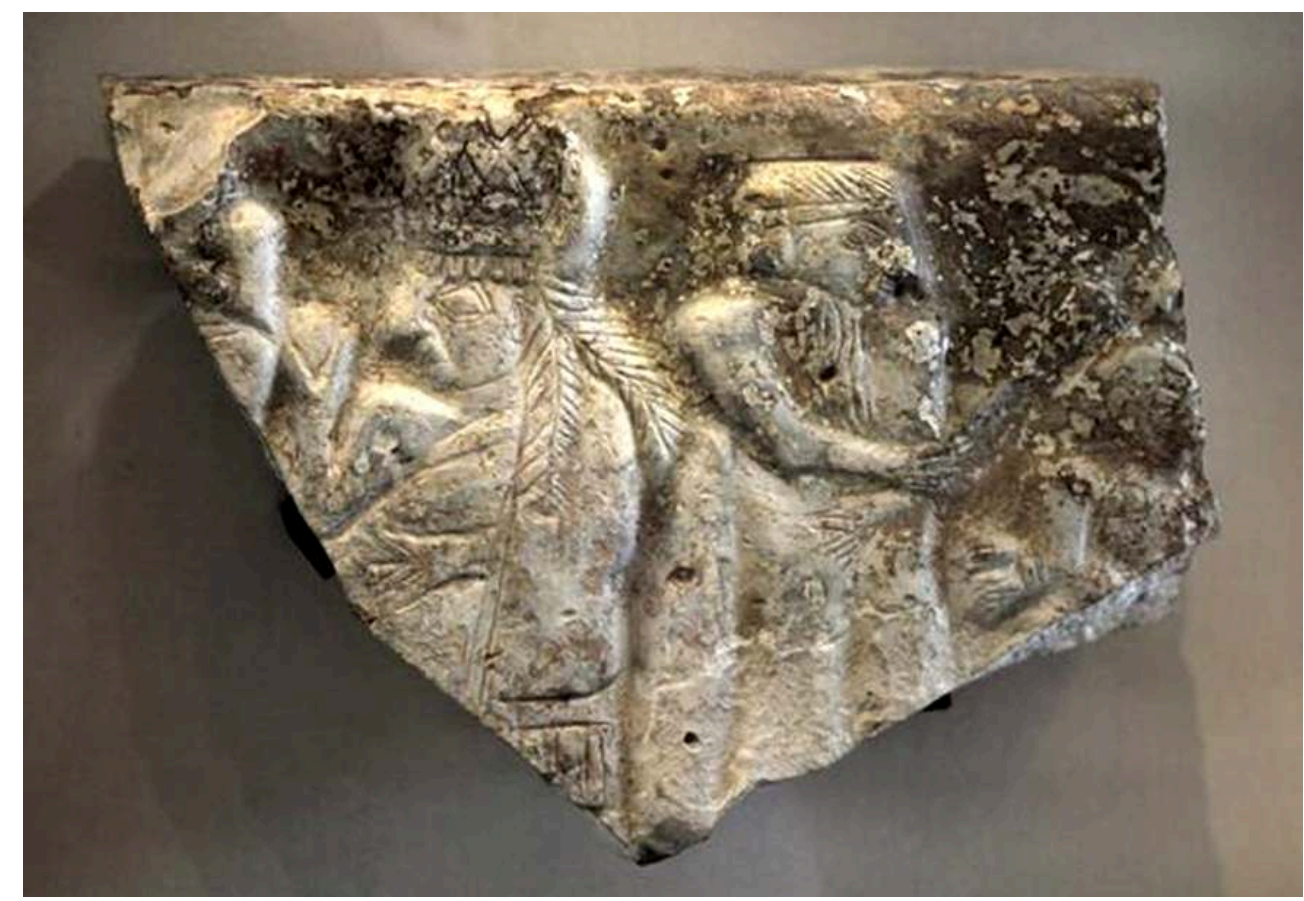

Tello, ca. 2600 av. J.-C. Musée du Louvre, Paris, AO 48. H : 18,5 cm.

Photo : Rama, Wikimedia Commons, CC BY-SA-2.0 FR. 
Fig. 2b : Vase en chlorite fragmentaire représentant Nisaba (?)

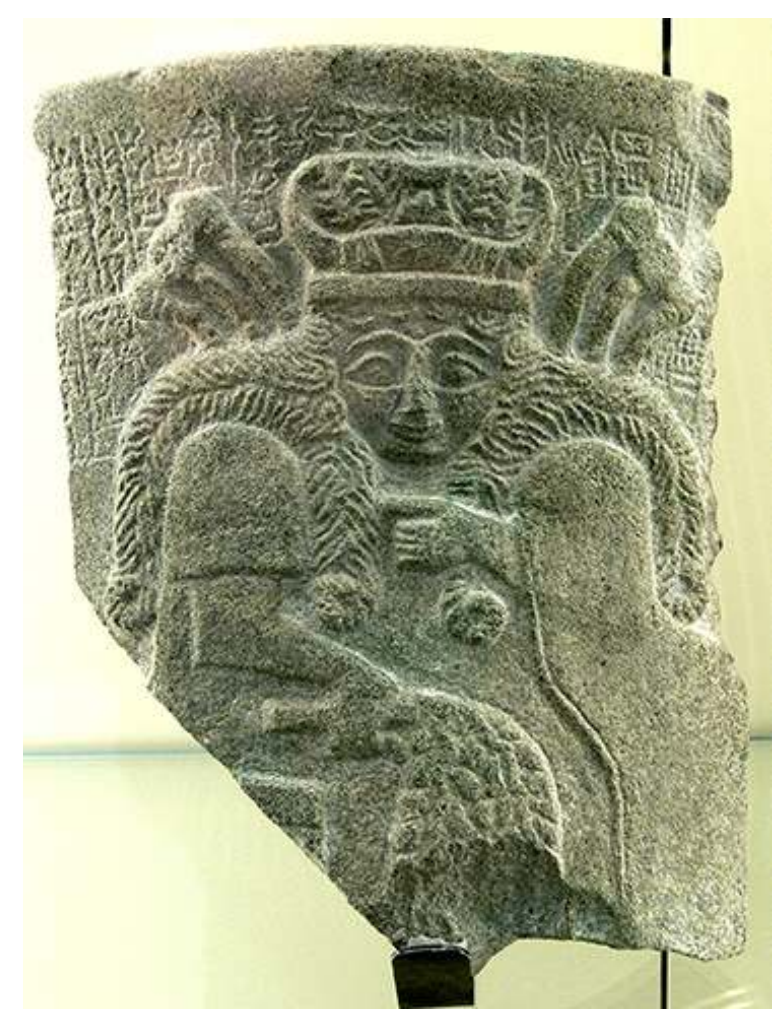

Lagash, ca. 2430 av. J.-C. Vorderasiatisches Museum, Berlin. H: $25 \mathrm{~cm}$. Photo: Wolfgang Sauber, Wikimedia Commons, CC BY-SA 3.0. 
Fig. 2c: Fragment de plaque perforée protodynastique représentant une déesse tenant des fuseaux.

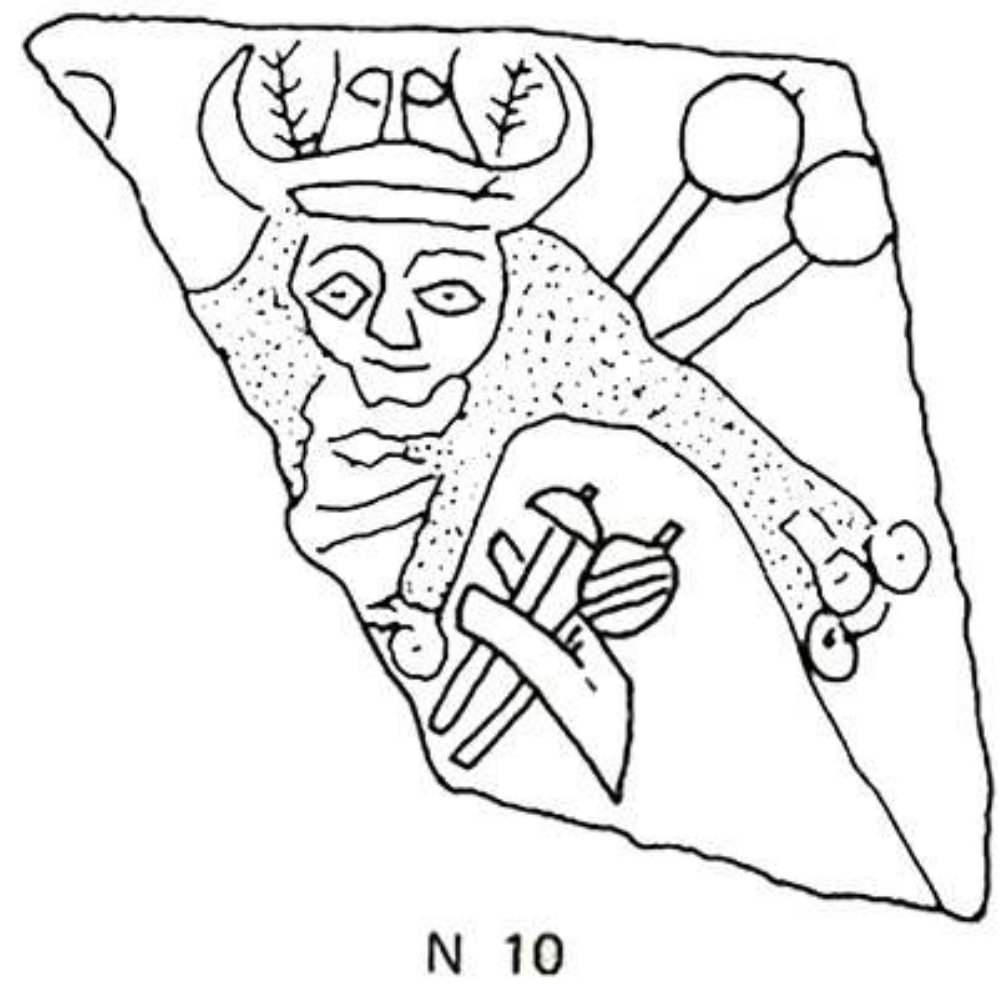

Nippur, première moitié du IIIe millénaire av. J.-C. H : $5 \mathrm{~cm}$.

D'après Boese 1971, nº 10.

Nombre de ces divinités semblent féminines mais cette observation ne peut être généralisée (Boese 1971, $n^{\text {os }} 8$ et 9 ; Asher-Greve et Westenholz 2013, p. 39). Elles apparaissent en position assise, associées à divers attributs, variables en fonction des scènes représentées, gobelet, rameau, masses d'armes (?), etc. Le caractère statique des images suggère que nous sommes face à des statues plus qu'à des êtres animés, mais la question fait encore débat (Bachelot et Joannès 2001, p. 787). En effet, il n'existe pas de terme pour désigner "l'art» au sens actuel du terme dans les langues orientales anciennes, seules des « images » investies d'une forme de vie par le rituel sont attestées (Winter 1992, p. 13 et 15 ; Benzel 2015). Cela nous permet d'envisager plus en détail leurs parures de tête.

\section{Premières coiffures divines : des ensembles composites}

9 Ces premières divinités présentent une tête qui est souvent montrée de face, surmontée d'une coiffure cornue spectaculaire agencée de façon complexe, dotée d'une abondante chevelure tombant sur les épaules, sur la poitrine et dans le dos, tandis que des sortes de crans réguliers matérialisent une "frange" sur le front. Des cheveux en fibres pouvaient être intégrés aux coiffes elles-mêmes. Le traitement des cheveux, lui, est plus difficile à cerner en raison de variations iconographiques (ondulations, hachures, ligne médiane) pouvant renvoyer à une ou plusieurs réalités : cheveux défaits séparés en deux ou trois mèches, cheveux tressés, cheveux emballés dans des bandes, boucles 
terminales, etc. Toutes ces options sont possibles. Dans certains cas, une partie des cheveux semble ramenée en chignon à l'arrière de la tête (Boese 1971, nos 8 et 11), le chignon étant maintenu par un serre-tête comme sur le casque du roi Meskamlamdug (ca. 2600 av. J.-C.). Cette variante est peut-être la plus attestée ou du moins, celle qui a connu la postérité la plus longue. Que la chevelure soit vraie ou postiche est un point qui ne trouve pas de réponse (Huot 1989, p. 194). On conviendra que les artistes ont cherché à contourner une difficulté majeure qui est celle de la représentation d'un être qui n'existe pas et qui, de surcroît, doit porter des cornes sortant le plus naturellement possible d'un crâne qui n'en est pas naturellement pourvu !

Depuis l'époque d'Uruk IV, les textes archaïques font état d'un signe MEN qui désigne quelque chose qui est posé sur la tête et que l'on traduit par couronne. Ce signe semble toujours associé à la sphère divine. Un autre signe AGA semble désigner un diadème ou serre-tête associé cette fois au domaine royal (Asher-Greve 1995-1996, p. 183). D'un point de vue lexical, l'opposition est nette, elle semble clairement mise en image sur le relief AO 48 du Louvre (fig. 2a). Mais c'est bien le seul exemple pour lequel cette lecture fonctionne. Essayons d'en déterminer les différents éléments car ces couronnes sont clairement composites (Furlong 1987, pour un essai assez inabouti de classification).

11 Faisant abstraction de la taille souvent réduite des représentations (de l'ordre de quelques centimètres) et d'une certaine variabilité des dessins (fig. 3b), on peut isoler :

12 - un premier support posé à même la chevelure, cerclage complet ou simple serre-tête, servant à maintenir les cheveux en place comme à supporter les éléments supérieurs ;

- une paire de cornes qui semble d'un seul tenant, présentant parfois un renflement central ;

- des éléments obliques assujettissant les cornes au support ;

- des éléments végétaux (?) stylisés disposés de part et d'autre d'un élément central trapézoïdal ;

- ce que l'on identifiera à un «cimier", parfois lui-même surmonté d'une paire de cornes ou d'un croissant (fig. 3a). 
Fig. 3a : Schéma montrant l'agencement des différents éléments des coiffes divines protodynastiques.

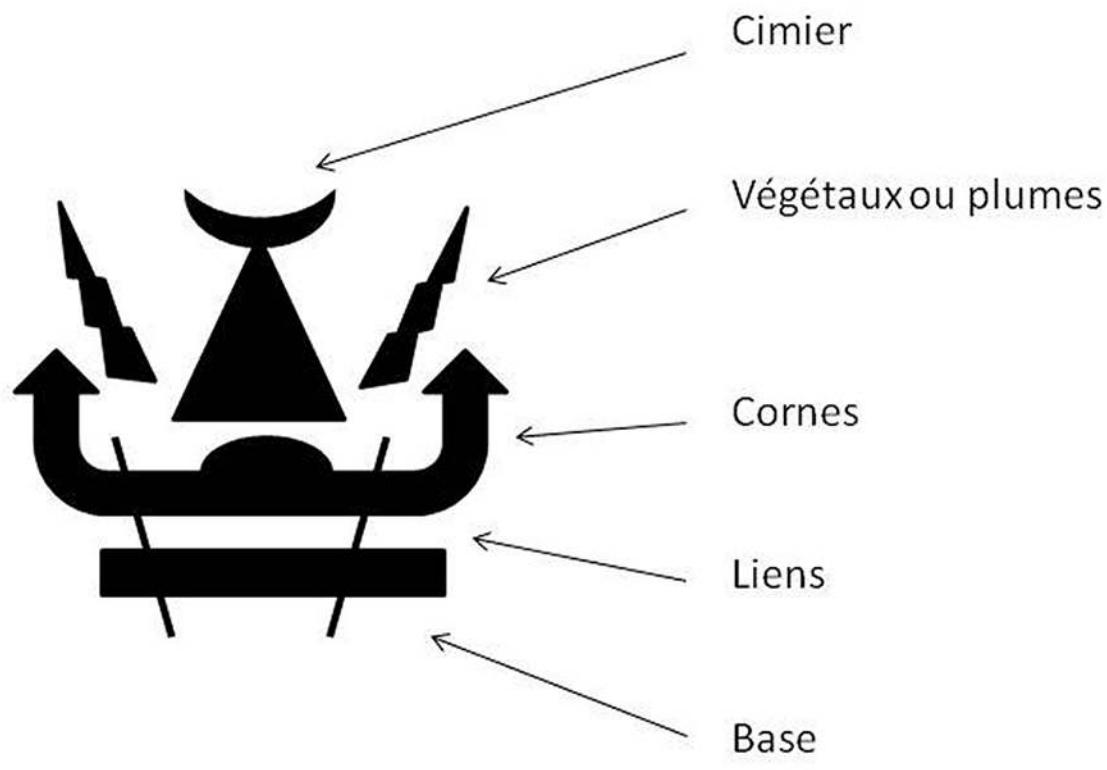

Dessin : Catherine Breniquet.

Fig. 3b : Détail des coiffures divines sur les sceaux-cylindres et vases en pierre protodynastiques.
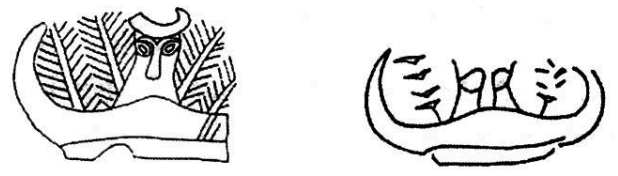

NiIr
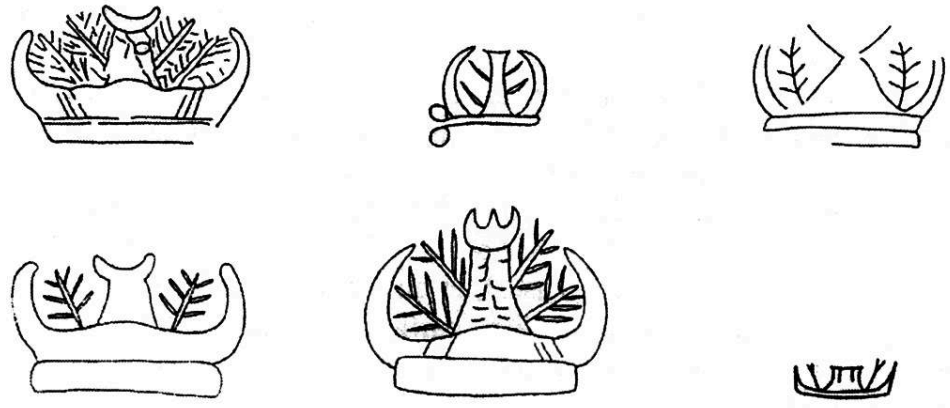

\section{UIT)}
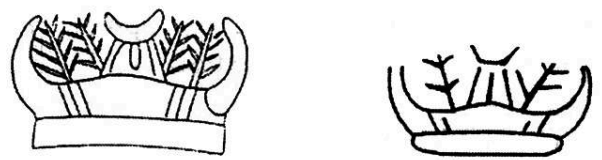

\section{L先}

Échelles diverses en fonction des objets originaux (de quelques $\mathrm{mm}$ à quelques $\mathrm{cm}$ ) D'après Asher-Greve 1995-1996, pl. 1, p. 189. 
Fig. 3c : Têtes de lion protodynastiques.
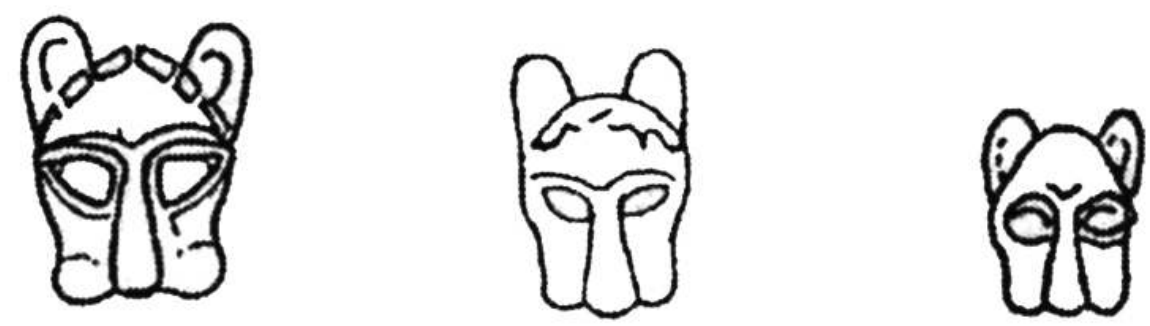

Échelles diverses (quelques $\mathrm{cm}$ )

D'après Asher-Greve 1995-1996, pl. 1, p. 189.

Fig. 3d : Stèle en gypse de Ninhursag.

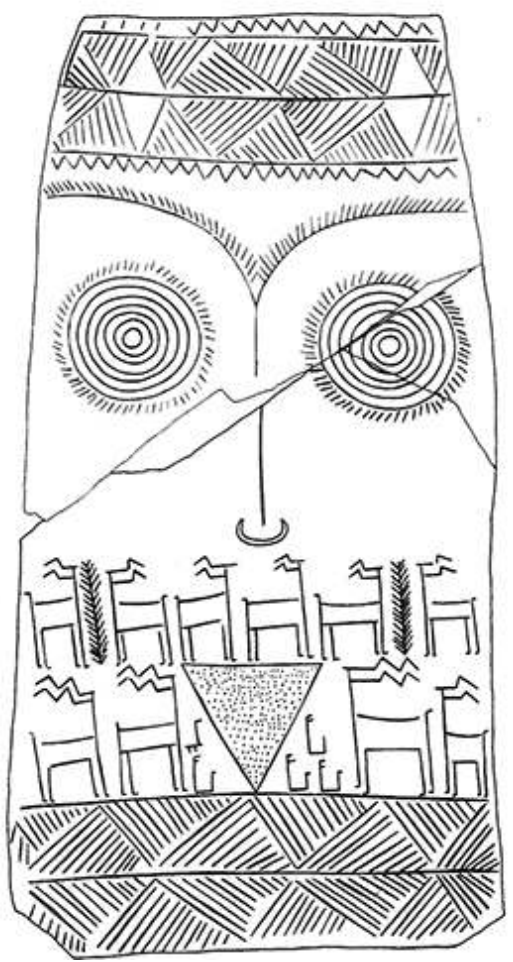

Mari, découverte dans la Ville II (2500-2250 av. J.-C.). H. : 35,7 cm.

D’après Margueron 2007, fig. 2, p. 126.

13 Ce cimier est presque toujours doté d'yeux surdimensionnés et d'un nez bien marqué (fig. 3c). Julia Asher-Greve (1995-1996) a proposé de l'identifier au masque stylisé d'un lion ${ }^{3}$ (par ailleurs attesté dans l'iconographie contemporaine, souvent associé à l'oiseau Anzu comme sur la stèle des Vautours ou le vase d'Enmetena), partant d'une analogie formelle simple. En première approche, l'assimilation est d'autant plus convaincante que les textes l'appuient. Ainsi les cornes évoqueraient la puissance de la divinité par analogie avec la force du taureau. Le lion, quant à lui, renverrait à la splendeur terrifiante de l'animal, et donc, à celle de la divinité. Dans les deux cas, cornes et masque léonin fileraient la métaphore animale. Toutefois, l'iconographie reste plus ou moins précise en fonction des supports et des objets et les détails sont parfois peu clairs 
ou très stylisés, de sorte que l'identification d'un masque léonin demeure très discutable. Le cimier prend parfois la forme schématique d'une "tour" crénelée, ou simplement triangulaire, doté d'un seul œil cyclopéen, assez éloigné dans les deux cas de la morphologie féline (fig. 2a). Les cornes sont parfois assimilées à un croissant disposé horizontalement. On peut penser que ce ne sont que des détails liés à l'absence de conventions artistiques fermement établies, mais il nous semble que l'on force ici la démonstration en s'appuyant sur les textes, au mépris du motif. L'exercice de description mérite d'être poussé plus en avant.

Aucune de ces coiffures n'étant connue directement par l'archéologie, le questionnement porte sur deux points : la taille réelle de l'ensemble composite et la nature des éléments qui le composent. En première approche et en supposant que l'iconographie est bien réaliste et ne fait pas varier les échelles sur une même composition, ne serait-ce que pour des raisons de lisibilité sur les objets minuscules que sont les sceaux-cylindres, la coiffure semble au moins égale à la hauteur de la tête, soit une bonne vingtaine de centimètres.

Les cornes apparaissent toujours de dimensions "normales" et en position anatomique. À cet égard, on peut suggérer qu'il s'agit de vraies cornes. Toutefois, rien n'oblige à imaginer qu'il s'agit d'une encornure extravagante qui évoquerait les cornes de l'auroch néolithique mis en scène sur les murs de Çatal Höyük par exemple, ou celles du bœuf à bosse ou encore du buffle ou du bison. Ces cornes semblent plutôt être celles d'un animal domestiqué, plutôt femelle d'ailleurs que mâle, en raison de leurs dimensions relatives. On pensera à une vache. Une observation plus attentive encore attire l'attention sur le dôme qui les relie de façon systématique et qui suggère que ce ne sont pas uniquement les cornes qui ont été rapportées, mais la partie supérieure de l'os frontal qui a dû être découpée pour confectionner la coiffe. Dès lors, on comprend mieux la présence des « liens », cordes ou cuir, qui les maintiennent à leur support, à la jonction du crâne et de la cheville osseuse. Que ces cornes aient été recouvertes d'un placage en métal précieux, or ou argent, est fortement plausible.

Les éléments stylisés, installés dans l'espace libre entre les cornes, nous semblent davantage correspondre à des plumes, si l'on en juge par le caractère réaliste de certaines représentations (fig. 3b). Toutefois, aucune codification systématique de ces éléments n'existe à cette époque. Une alternative pourrait être l'identification comme rameaux végétaux, mais elle n'apporte pas davantage de certitude.

17 Le cimier, enfin, s'écarte du masque léonin par son allure nettement trapézoïdale, son aspect anthropomorphe et ses cornes qu'on ne saurait réduire à des oreilles. À notre connaissance, aucun lion cornu n'est attesté dans l'iconographie de cette époque. On connaît quelques têtes de lion en relief ou ronde-bosse (lions en bronze du temple « aux lions » de Mari, têtes de lion du temple de Ninhursag à Tell el'obeid, placages du traineau de Pu-abi à Ur), mais aucune d'entre elles ne paraît correspondre à une telle fonction de cimier. Toutes sont bien rondes et joufflues et leur destination ne présente aucune ambiguïté. On en arrive ainsi à remettre en question l'interprétation proposée par J. Asher-Greeve.

\section{Le cimier}

Quelle peut être alors la nature de l'élément central des coiffures divines ? D'après les caractéristiques récapitulées ci-dessus, le seul objet archéologique connu qui pourrait 
être en adéquation avec celles-ci, est la "stèle de Ninhursag " découverte dans les niveaux protodynastiques de Mari (Margueron 2007) (fig. 3d). Cette dernière s'intègre dans une petite série qui comprend plusieurs fragments similaires trouvés sur ce même site. Je propose que ces objets soient des cimiers de coiffures divines protodynastiques. La stèle de Ninhursag offrirait l'exemplaire complet le plus spectaculaire. Plusieurs arguments appuient l'hypothèse : la forme générale, trapézoïdale, l'accent mis sur le haut du visage et plus particulièrement les yeux, la coiffure tressée ménageant deux " cornes ", la base dont les angles ont été coupés pour être insérés dans un support, le contexte enfin, à savoir un dépôt dans une petite fosse du temple dit de Ninhursag à Mari, dont la complexité stratigraphique avait été repérée dès les premières investigations sur le terrain (Parrot 1940 ; Beyer et Jean-Marie 2007). L'objet y avait été déposé (et non jeté) avec d'autres, cassé et réparé dans l'Antiquité (Margueron 2007, p. 123). Découvert dans la Ville II, il pourrait être plus ancien et dater de la Ville I. Aucun doute ne peut exister sur le caractère exceptionnel de l'objet.

L'interprétation comme stèle doit rester descriptive (au sens de dalle gravée et dressée) car l'objet a été découvert en contexte secondaire, sans aucun autre élément permettant de préciser une destination votive. Or, il pourrait être un élément d'un tout composite, en l'occurrence une coiffe divine spectaculaire, dont la taille imposante $(36 \mathrm{~cm}$ de hauteur) suggère une statue monumentale, double de la taille humaine. Rapportée aux dimensions d'un crâne de vache, l'hypothèse est cohérente et plausible, et est en outre confortée par ce que montre l'iconographie où les dieux assis sont environ deux fois plus grands que les mortels.

Récapitulons. Ces premières parures de têtes des divinités mésopotamiennes sont donc bien étranges et échappent à toute lecture illustrative immédiate. Il est impossible au premier coup d'œil de savoir à quoi on a affaire et plus encore, de faire l'adéquation avec un mot. Dépassons un constat aussi affligeant. Pour autant qu'on puisse en juger, ces parures de tête sont composites et associent des éléments naturels (os de bovin, liens en cuir ou cordes, plumes ou végétaux) qui ont disparu du fait de leur nature périssable ou recyclable, et d'autres, manufacturés comme le cimier, seul élément potentiellement attesté par l'archéologie. Le masque anthropomorphe qui le constitue échappe aussi à toute classification simpliste. On s'accorde à dire qu'il évoque un visage, mais cette description est notoirement insuffisante. On rappellera que les représentations dont on dispose sur la glyptique sont trop petites et trop stylisées pour permettre une identification satisfaisante et systématique des détails (fig. 3b). Ce " masque » est tout à la fois visage et corps (les yeux pouvant évoquer la poitrine, et la bouche, le sexe féminin) et n'aurait pas été désavoué par les artistes contemporains comme Magritte, mais il est aussi masculin et féminin avec la présence d'une possible barbe. Il associe en outre le monde animal avec les cervidés (?), les oiseaux et le monde végétal (avec les rameaux stylisés), et est humain et divin (en raison de la présence de possibles cornes dans les cheveux tressés).

Plusieurs auteurs insistent sur la parenté de la stèle de Ninhursag avec d'autres objets trouvés dans des régions éloignées, Levant et monde ibérique, dans des contextes plus anciens, $\mathrm{VI}^{\mathrm{e}}$ et $\mathrm{V}^{\mathrm{e}}$ millénaires (Milevski et al. 2016, Schuhmacher 2013, Uehlinger 2014). Les objets en question sont de nature diverse: plaques gravées en pierre, os longs gravés, vases, etc. Le motif des yeux grands ouverts en est l'ornementation principale. Une observation attentive permet de constater qu'il n'est associé au reste d'un visage ou à des animaux et des végétaux que dans certains cas seulement. Les auteurs 
mentionnés insistent sur les interactions culturelles entre les régions concernées susceptibles d'être mise en lumière par la diffusion de ce motif anthropomorphe à partir du Levant (Milevski et al. 2016, p.146). La chronologie de l'ensemble du mouvement est encore imprécise mais s'effectuerait sur la « longue durée » (Uehlinger 2014). Il est peut-être périlleux de tirer trop de conclusions diffusionnistes de ces parallèles, sauf à conclure comme Thomas Schuhmacher (2013, p. 17) que l'émergence du motif pourrait être liée à l'implantation - en Espagne - d'un atelier de travail de l'ivoire en provenance de Méditerranée. Pour beaucoup d'artefacts, c'est bien le motif des yeux qui a été privilégié. On est en droit de se demander si celui-ci ne présente pas un caractère universel, susceptible d'apparaître ou de réapparaître de façon autonome en plusieurs endroits. À cet égard, les vases en pierre de l'époque d'Uruk (Basmachi 1975-1976, n ${ }^{\circ} 35$ ) ou la glyptique d'Iran ou de la Diyala montrant des yeux plus ou moins stylisés (Amiet 1980, pl.18, $\mathrm{n}^{\text {os }} 292,295,468,480,501$ et 681 ) complètent ces séries sans être mentionnés dans les possibles comparaisons. Quoi qu'il en soit, les séries du Levant (Hagoshrim, Neve Yam et Ein Zippori surtout) et d'Aššur (Milevski et al. 2016) offrent sans conteste les parallèles les plus convaincants, à un détail près : ces objets sont minuscules par rapport à la stèle de Ninhursag. Au mieux, ils pourraient correspondre à de lointains prototypes élaborés par des communautés agraires à la fin du Néolithique sur une zone géographique vaste, au Proche-Orient. À l'appui de cette hypothèse, on notera que les plaquettes d'Aššur semblent plus anciennes que leur contexte de découverte : elles sont peut-être néolithiques (Milevski et al. 2016, p. 143).

Le découvreur de la stèle, Jean-Claude Margueron, alors directeur de la mission de Mari (Margueron 2007), ainsi que d'autres commentateurs (Schuhmacher 2013, Uehlinger 2014) ont insisté sur le fait qu'on aurait là une possible évocation de Ninhursag, la " Dame de la Montagne », la déesse-mère sumérienne, une des plus anciennes divinités connues, créatrice, mère de tout ce qui vit, mentionnée dans les textes sans doute dès l'époque d'Uruk (ca 3900-3000), mais de façon certaine au Protodynastique I (2900-2800). Cette constatation ne fait pas pour autant de la divinité qui porte cette parure de tête la représentation de Ninhursag elle-même (ou du moins pas de façon systématique). La prudence reste de mise car, au jeu de l'analogie, on pourrait aussi reconnaître une chouette (attestée sous forme de figurine dès Mureybet III : Cauvin 1978, fig. 26-2, p. 122) et ainsi glisser vers un autre registre interprétatif. Il ne resterait alors pas grand-chose de Ninhursag... Un tel cimier peut évoquer un pouvoir suprême dont nous ignorons tout, une sorte de référence à une lointaine divinité originelle dont celui ou celle qui le porte tirerait avantage.

Les autres détails iconographiques sont également sujets à discussion. Les cornes notamment pourraient tout autant évoquer une divinité nourricière assimilée à une vache, que la force brute du taureau sauvage. Le double argument serait que l'on ne s'accorde pas sur le sens des bucranes néolithiques (culte du taureau, symboles de prestige ou évocation de la société) (Testart 2006; Forest et Gallois 2006, p. 24), et que l'idéologie des premières sociétés agraires met volontiers en scène la vache comme animal originel. À titre d'exemples, on citera la représentation de la déesse égyptienne Hathor, portant une coiffure cornue entourant le disque solaire car la forme première de cette divinité était une vache, ou dans le domaine de l'ethnographie, les coiffures féminines des femmes Khalkha, en Mongolie, avec des chevelures postiches formant deux « cornes » qui renvoient explicitement à celles de la vache mythique originelle de leur système de pensée (Bromberger 2010, p.11). Nous ne disposons pas de tels éclairages pour la Mésopotamie à l'époque qui nous concerne, mais on peut citer 
l'incantation akkadienne mettant en scène Geme-Sîn, la vache favorite du dieu Lune Sîn, enceinte de ses œuvres. Entendant ses cris lors de la mise-bas, Sîn lui envoya deux créatures chargées d'accomplir un rituel facilitant la naissance. L'histoire sert de support à une supplique pour que les femmes donnent la vie aussi facilement que Geme-Sîn (Veldhuis 1991). À plus basse époque encore, à l'époque assyrienne, c'est Ishtar de Ninive qui est comparée à une vache, nourrice du roi d'Assyrie (Parpola 1997, p. XXXVI-XLII). Comme dans l'Égypte ancienne, cette métaphore serait traduite dans l'iconographie sous les traits d'une vache allaitant son veau (Keel 2013) ${ }^{4}$. L'ensemble de ces observations fait ainsi sens à un niveau d'abstraction qui dépasse la matérialité de l'archéologie.

Enfin, les feuillages ou plumages entourant les cimiers de ces coiffures, pourraient sans peine symboliser ou matérialiser l'union avec les autres mondes vivants.

\section{Couronnes divines, coiffures des mortels}

L'observation de la coiffure de la stèle de Ninhursag avec ses deux (?) mèches tressées terminées par des cornes renvoie aussi aux autres coiffures contemporaines, celles des mortels. Les représentations protodynastiques de femmes, toutes issues de l'élite de la société, montrent qu'elles portaient les cheveux longs, mais rassemblés en chignons compliqués, d'où sortaient une, voire deux, longues nattes enroulées autour de la tête (Frankfort 1939, pl. 89 et 90 ; Spycket 1954 et 1955). Les coiffures masculines invitent à prolonger ces observations. Quand ils n'ont pas le crâne rasé, les hommes portent ces coiffures à chignon, avec deux mèches tressées, ramenées sur le devant du crâne. Il est étonnant que ces deux tresses n'aient jamais été vues comme de possibles cornes et donc, comme une allusion à la sphère divine. Cette coiffure est semble-t-il aussi associée à l'univers royal (casque de Meskalamdug, ca. 2600, tête en bronze dite de Sargon d'Akkad trouvée à Ninive, ca. 2250, par exemple) (Basmachi 1975-1976, nos 84 et 103). Elle apparait aussi dans le monde divin, comme l'atteste la face mythologique de la Stèle des Vautours (ca.2450) montrant le dieu Ningirsu coiffé de la sorte. Une malencontreuse cassure a toutefois fait disparaître sa couronne, ce qui ne permet pas de l'identifier à coup sûr sur cette seule base. Ce mode d'agencement des cheveux apparaît en demi-teintes, glissant du répertoire divin au répertoire royal ou l'inverse, de façon plus subtile qu'on veut bien le dire. D'un point de vue iconographique, il fonctionne comme une mise en abîme.

\section{Perspectives}

L'ensemble de ces observations nous renvoie semble-t-il à des conceptions du divin sans lien avec ce qui existe aux époques ultérieures en Mésopotamie. Ce qui est en jeu est la nature "formative", primaire, originelle, des périodes sur lesquelles le propos est focalisé. Elles constituent un moment que nous peinons à saisir à travers nos sources, celui de la mise en place des premières divinités de Mésopotamie, évoquées au moyen d'attributs originels. La stèle de Ninhursag renvoie peut-être à cette puissance des origines. On pourrait en dire de même de tous les attributs " créateurs ", attestés en d'autres endroits du monde, comme les objets liés au travail du fil, quenouille et fuseau par exemple (Boese 1971, $\mathrm{n}^{\circ} 10$; Scheid et Svenbro 2003 ; Breniquet 2008, p. 373-380) (fig. 2c). Leur forme, simple et prêtant à confusion dans l'iconographie, ne nous permet 
pas toujours de les identifier avec certitude, on y voit plus volontiers des masses d'armes, conformément à l'iconographie mésopotamienne plus récente ainsi qu'au paradigme de lecture sous-jacent. Nos sources sont en cause, mais nos traditions de recherche aussi. Sans vouloir prôner une démarche a-historique faite de grandes généralités, un rapprochement avec d'autres branches des sciences humaines s'impose : ethnographie, anthropologie culturelle, sciences cognitives, etc. (Pongratz-Leisten et Sonik 2015).

\section{BIBLIOGRAPHIE}

Amiet P. (1977), « Pour une interprétation nouvelle du répertoire iconographique de la glyptique d'Agadé », Revue d'assyriologie et d'archéologie orientale 71-2, p. 107-116.

Amiet P. (1976), L'art d'Agadé au musée du Louvre, Paris.

Amiet P. (1980), La glyptique mésopotamienne archaïque, Paris.

Asher-Greve J. (1995-1996), « Reading the Horned Crown: A Review Article », Archiv für Orientforchung 42-43, p. 181-189.

Asher-Greve J. et Westenholz J. (2013), Goddesses in Context: On Divine Powers, Roles, Relationships and Gender in Mesopotamian Textual and Visual Sources, Fribourg-Göttingen.

Bachelot L. et Joannès F. (2001), « Statues de culte ", dans Joannès F. éd., Dictionnaire de la civilisation mésopotamienne, Paris, p. 787-789.

Basmachi F. (1975-1976), Treasures of the Iraq Museum, Baghdad.

Becker A. (1993), Uruk. Ausgrabungen in Uruk-Warka: Endberichte 6, Kleinfunde I, Berlin.

Benzel K. (2015), « 'What Goes in Is What Comes Out' - But What Was Already There? Divine Materials and Materiality in Ancient Mesopotamia ", dans Pongratz-Leisten B. et Sonik K. éd., The Materiality of Divine Agency, Berlin, p. 89-118.

Beyer D. et Jean-Marie M. (2007), « Le temple du DA III de la déesse Ninhursag à Mari : les dépôts votifs du Lieu Très Saint », Akh Purattim 2, p. 75-122.

Black J. et Green A. (1992), Gods, Demons and Symbols of Ancient Mesopotamia: An Illustrated Dictionary, London.

Boehmer R. M. (1967), « Die Entwicklung der Hörnerkrone von ihren Anfängen bis zum Ende der Akkad-Zeit », Berliner Jahrbuch für Vor- und Frühgeschichte 7, p. 273-291.

Boehmer R. M. (1971), « Hörnerkrone », Reallexikon der Asyriologie 4, p. 431-434.

Boese J. (1971), Atmesopotamische Weihplaten, Berlin.

Breniquet C. (2008), Essai sur le tissage en Mésopotamie, des premières communautés sédentaires au milieu du III millénaire avant J.-C., Paris.

Bromberger C. (2010), Trichologiques, une anthropologie des cheveux et des poils, Paris.

Cauvin J. (1978), Les premiers villages de Syrie-Palestine, du IXe au VII millénaire avant J.-C., Lyon. 
Collins P. (1994), « The Sumerian Goddess Inanna (3400.2200 BC) », Papers from the Institute of Archaeology 5, p. 103-118.

Conteneau G. (1952), Le Déluge babylonien, Ishtar aux Enfers, la Tour de Babel, Paris.

Coquet M. (1994), « L'envers du regard », Journal des africanistes 64-II, p. 39-63.

Englund R. (1998), « The Texts from the Late Uruk Period », dans Bauer J., Englund R. et Krebernik M. éd., Mesopotamien Späturuk-Zeit und Frühdynastiscghe Zeit, Fribourg-Göttingen, p. 15-233.

Falgayrettes-Leveau C. (2003), Parures de tête, Paris.

Forest J.-D. (1996), Mésopotamie. L'apparition de l'État, Paris.

Forest J.-D. et Gallois N. (2006), « L'art mésopotamien. Architecture et arts plastiques des origines à la fin du IVe millénaire ", dans Curatola G. dir., L'art en Mésopotamie, Paris, p. 15-64.

Frankfort H. (1939), Sculpture of the Third Millennium from Tell Asmar and Khafajah, Chicago.

Furlong I. (1987), Divine Headdresses of Mesopotamia in the Early Dynastic Period, Oxford.

Gell A. (1998), Art and Agency. An Anthropological Theory, Oxford.

Huot J.-L. (1989), Les Sumériens. Entre le Tigre et l'Euphrate, Paris.

Huot J.-L. (2005), « Vers l'apparition de l'État en Mésopotamie : bilan des recherches récentes », Annales. Histoire, Sciences Sociales 60-5, p. 953-973.

Keel O. (2013), « Das Böcklein in des Milch seiner Mutter und Verwandtes, im Lichte eines altorientalischen Bildmotivs ", dans Lippke F. éd., V. IRAT III-KEE 1980.1 (Böcklein), JerusalemTübingen.

Le Breton D. (1992), Des visages. Essai d'anthropologie, Paris.

Lindemeyer E. et Martin L. (1993), Uruk Kleinfunde III, Mainz am Rhein.

Margueron J.-Cl. (2007), « Une stèle du temple dit de Ninhursag », Akh Purattim 2, p. 123-134.

Milevski I., Getzov N., Galili E., Yaroshevich A. et Kolska Horwitz L. (2016), « Iconographic Motifs from the 6 th $-5^{\text {th }}$ millennia BC in the Levant and Mesopotamia: Clues for Cultural Connections and Existence of an Interaction Sphere ", Paléorient 42-2, p. 135-149.

Nöldeke A. et Lenzen J. (1940), UVB 11 : Die Kleinfunde, Berlin.

Oppenheim L. (1970), La Mésopotamie. Portrait d'une civilisation, Paris.

Parpola S. (1997), Assyrian Propheties, Helsinki.

Parrot A. (1940), « Les fouilles de Mari », Syria 21, p. 1-28.

Pongratz-Leisten B. et Sonik K. (2015), « Between Cognition and Culture: Theorizing the Materiality of Divine Agency in Cross-Cultural Perspective ", dans Pongratz-Leisten B. et Sonik K. éd., The Materiality of Divine Agency, Berlin, p. 3-69.

Scheid J. et Svenbro J. (2003), Le métier de Zeus. Mythe du tissage et du tissu dans le monde grécoromain, Paris.

Schuhmacher T. (2013), « Some Reflections about an Alabaster Stele from Mari (Syria) and Its Possible Relations to the Western Mediterranean ", Cuadernos de Prehistoria y Arqueología de la Universidad Autónoma de Madrid 39, p. 7-20. 
Spycket A. (1954), « La coiffure féminine en Mésopotamie : des origines à la $1^{\text {ère }}$ dynastie de Babylone ", Revue d'assyriologie et d'archéologie orientale 48-3, p. 113-129.

Spycket A. (1955), « La coiffure féminine en Mésopotamie : des origines à la $1^{\text {ère }}$ dynastie de Babylone (suite et fin) ", Revue d'assyriologie et d'archéologie orientale 49-3, p. 113-128.

Spycket A. (1968), Les statues de culte dans les textes mésopotamiens des origines à la I İre dynastie de Babylone, Paris.

Testart A. (2006), « Interprétation symbolique et interprétation religieuse en archéologie. L'exemple du taureau à Çatal Höyük », Paléorient 32-2, p. 23-57.

Uehlinger C. (2014), « NinHुursaĝa oder „Große Mutter"? Eine ikonographisch-ikonologische Skizze zu einem Phänomen der longue durée », dans Wimmer S. et Gafus G. éd., Vom Leben umfangen Ägypten, das Alte Testament und das Gespräch der Religionen Gedenkschrift für Manfred Görg, Münster, p. 407-424.

Veldhuis N. (1991), A Cow of Sin, Leiden.

Winter I. (1992), « 'Idols of the Kings'. Royal Images as Recipients of Ritual Action in Mesopotamia ", Journal of Ritual Studies 6-1, p. 13-42.

\section{NOTES}

1. Je tiens à remercier très chaleureusement Florence Gherchanoc et Louise Quillien pour leur invitation et leur aide, ainsi que Béatrice Muller et Jean-Claude Margueron pour les échanges scientifiques autour de la stèle de Ninhursag de Mari. Naturellement, l'interprétation proposée n'engage que moi.

2. Je laisse volontairement de côté le cas du Vase d'Uruk, maintes fois réparé, précisément au mauvais endroit, celui de la coiffure du personnage féminin du registre supérieur (Lindemeyer et Martin 1993, Taf. 21).

3. On notera que la crinière n'est pas clairement représentée, ce qui tend à faire de l'animal une lionne.

4. Je remercie Cécile Michel et Pierre Villard pour m’avoir signalé ces références.

\section{RÉSUMÉS}

Tout au long de l'histoire de l'Orient ancien, les divinités ont porté des coiffures cornues, diadèmes, caches-cheveux, casques, tiares à un ou plusieurs rangs de cornes. On étudiera les représentations des parures de tête des premières divinités connues en Mésopotamie, aux époques pré- et protodynastiques (IV millénaire-première moitié du $\mathrm{III}^{\mathrm{e}}$ millénaire av. J.-C.). À travers l'iconographie, il est possible d'avoir une idée concrète de l'aspect matériel des coiffes de ces premières divinités. Elles formaient des ensembles composites originaux associant des éléments naturels et manufacturés, dont un masque anthropomorphe à la symbolique énigmatique. L'un d'eux pourrait correspondre à la prétendue « stèle de Ninhursag » de Mari. 
In the long history of the Ancient Near East, deities wore horned headgear, diadems, haircuts, helmets, tiaras with one or more rows of horns. The purpose of this paper is to study the headdresses of the first known images of Mesopotamian deities, during the pre and protodynastic periods (4th millennium-first half of the 3rd millennium B.C.). Through iconography, it is possible to have a concrete idea of the material aspect of the headdresses of these early deities. They formed unsual composite ensembles combining natural and manufactured elements, where an anthropomorphic mask carrying an enigmatic value is embedded. We suggest that the stela of Ninhursag from Mari could be part of them.

INDEX

Keywords : Mesopotamia, goddess, hairdresses, horns, Ninhursag, Mari

Mots-clés : Mésopotamie, divinités, coiffures, cornes, Ninhursag, Mari

\section{AUTEUR}

\section{CATHERINE BRENIQUET}

Université Clermont Auvergne 